\title{
Total photoionization cross section of planer helium: scaling laws and collision orbits
}

\author{
Min-Ho Lee, Chang Woo Byun, Nark Nyul Choi \\ School of Liberal Arts and Teacher Training, Kumoh National Institute of \\ Technology, Gumi, Gyeongbuk 730-701, Korea.

\section{Gregor Tanner} \\ School of Mathematical Sciences, University of Nottingham, University Park, \\ Nottingham NG7 2RD, UK.
}

1 August 2018

\begin{abstract}
The total photoionization cross section of planar helium has been calculated up to the single ionization threshold $I_{22}$ of triple $P$ states. The cross section shows chaotic fluctuations as the energy $E$ approaches the double ionization threshold $E=0$. By analyzing the fluctuating part of the cross section, we show that its amplitude decreases as $|E|^{\mu}$ for $E \rightarrow 0^{-}$as predicted in [C. Byun et al., Phys. Rev. Lett. 98, 113001 (2007)]. The Fourier transform of the fluctuating part reveals peaks at the classical actions of closed triple collision orbits. Furthermore, the relative height of the peaks are consistent with the semiclassical predictions. Our findings underline that the fluctuating part of the photoionization cross section can be described by classical triple collision orbits in the semiclassical limit. These orbits all lie in the collinear eZe subspace, demonstrating that the fluctuations are dominated by the dynamics of this low dimensional phase space.
\end{abstract}

PACS numbers: 32.80.Fb,03.65.Sq,05.45.Mt,05.45.-a

Keywords: total photoionization cross section, scaling law, planar helium

Submitted to: J. Phys. B: At. Mol. Phys.

\section{Introduction}

Since Madden and Codling observed auto-ionization of helium from the ground state to doubly excited state in 1963 [1], two-electron atoms have served as a prototype for understanding electron-electron correlations in atomic systems. To describe two-electron atoms fully quantum mechanically, many efficient numerical methods, such as exterior complex scaling [2, 3], convergent closed coupling methods [4, 5], time-dependent closecoupling method $[6,7,8,9,10]$ or advanced hyperspherical R-matrix techniques using semiclassical outgoing waves $[11,12,13]$, have been applied. 
Total photoionization cross section of planer helium: scaling laws and collision orbits 2

In recent years, the photoionization cross section has been fully explored experimentally up to the $N \approx 15$ ionization threshold by virtue of the experimental advances in synchrotron radiation sources and detector technology $[14,15]$. However, only the spectrum of the bound states and the low-lying doubly excited resonances is well understood, see for example Ref. [11] for an overview. Furthermore, as one approaches the double ionization threshold $E=0$ from below, the photoionization cross section shows a highly chaotic behavior in the region of overlapping Rydberg series [11]. In Refs. [16, 17], based on the extended semiclassical closed orbit theory, it is shown that this behavior is intricately linked to the complexity of the underlying classical dynamics of the three-body Coulomb problem helium. In particular, the paper predicts two semiclassical results: (i) the Fourier spectrum of the fluctuating part of the photoionization cross section shows distinct peaks at the positions of the actions of socalled closed triple collision orbits (CTCO), that is, classical trajectories of the coupled two-electron dynamics starting and ending in the nonregularizable triple collision; (ii) the amplitude of the fluctuations in the total and partial cross sections for single electron photoionization in two-electron atoms decays algebraically in terms of a threshold law $|E|^{\mu}$ as $E \rightarrow 0$, where the exponent $\mu$ is given as

$$
\mu=\frac{1}{4} \operatorname{Re}\left[\sqrt{\frac{100 Z-9}{4 Z-1}}+2 \sqrt{\frac{4 Z-9}{4 Z-1}}\right]
$$

with $Z$, the charge of the nucleus. The exponent is related to the stability exponents of the CTCO's and differs from Wannier's exponent [18] dominating double ionization processes. The prediction implies that the photoionization cross section can be described semiclassically in terms of classical triple collision orbits all lying in the collinear eZe space.

By numerically calculating the total photoionization cross section (TPICS) of collinear eZe helium up to the 50th single ionization threshold, $I_{50}$, the semiclassical predictions have been confirmed in Refs [16, 17]. For fully three dimensional helium, however, a verification of these semiclassical results based on numerical computations or experiments has been missing so far. By analysing the experimental data in [14] on partial photoionization cross sections (PPICS) for helium up to $N \approx 13$, it could be shown in [19] that the fluctuations in the PPICS are semiclassically dominated by the contributions from closed triple collision orbits. The threshold laws could, however, not be extracted due to lack of data. In Ref. [15], Jiang et. al. have measured the total photoionization cross section up to single ionization threshold $I_{15}$ of $\mathrm{He}^{+}$and computed the spectrum up to $I_{17}$ numerically by using the complex rotation method [20]. It was shown that the spectra are dominated by principal Rydberg series, however, semiclassical results could not been extracted due to lack of resolution.

For planar helium, the fluctuating part of TPICS have been calculated up to the 20th single ionization threshold of triplet $P$ states using the complex rotation method [21]. The authors of [21] argued that the fluctuations in the low energy region are mainly due to a dominant series of resonances associated with an approximate quantum number 
Total photoionization cross section of planer helium: scaling laws and collision orbits 3

$F=N-K$, related to the collinear eZe dynamics. But as the energy increases, new series start to contribute significantly to the cross section, and it is suggested that the scaling law starts to become invalid. This is in contrast to findings in $[16,17]$, where it is predicted that planar helium should also follow the scaling law and other semiclassical results stated above.

Based on ab initio numerical calculation for the total photoionization cross section of planar helium and by employing a quantum map approach [16, 17], we will show in this paper, that the above mentioned semiclassical results are indeed valid. The paper is organized as follows. In Sec. II, we will briefly describe the semiclassical theory and the numerical implementation of the ab initio method. In Sec. III, the numerical result of the photoionization cross section of the planar helium up to $I_{22}$ is presented. By analysing the numerical data, the semiclassical results are confirmed for the first time in higher dimensions. Sec. IV concludes the paper. Unless stated otherwise, the atomic units are used throughout this paper.

\section{Theory}

\subsection{A Quantum map approach to the total photoionization cross section}

In the Poincare map approach introduced in [17], one starts from writing the photoionization cross section $\sigma(E)$ in terms of the retarded Green function $G(E)$,

$$
\sigma(E)=-\frac{4 \pi}{c} \omega \operatorname{Im}\left\langle D \phi_{0}|G(E)| D \phi_{0}\right\rangle
$$

Here, $\phi_{0}$ is the wave function of the initial state and $D=\boldsymbol{\epsilon} \cdot\left(\mathbf{r}_{1}+\mathbf{r}_{2}\right)$ is the dipole operator with $\boldsymbol{\epsilon}$, the polarization of the incoming photon and $\mathbf{r}_{i}$, the position of $i$-th electron. The direction of the polarization is taken along the $z$-axis.

Partitioning the whole configuration space into inner and outer regions by introducing a dividing surface $\Sigma$, we can express the cross section $\sigma(E)$ in terms of the local scattering matrices $\mathbf{r}, \mathbf{s}$ and the scattering solutions of the half-space Hamiltonians related to the inner and outer regions as

$$
\sigma(E)=\sigma_{b g}+\sigma_{f l}=\frac{4 \pi^{2}}{c} \omega\left[\mathbf{d}_{o}^{\dagger} \mathbf{d}_{o}+2 \operatorname{Re}\left[\mathbf{d}^{\dagger}(\mathbf{1}-\mathbf{r s})^{-1} \mathbf{r d}^{*}\right]\right]
$$

where $\Sigma$ is taken here at a fixed hyperradius $R=R_{0}$ with $R=\sqrt{r_{1}^{2}+r_{2}^{2}}$. Furthermore, $\mathbf{d}=\left\langle\Psi^{+}|D| \phi_{0}\right\rangle$ with $\Psi^{+}(E)$, the solutions of the Schrödinger equation $\left(H^{I}-E\right) \Psi=0$ satisfying outgoing boundary condition, where $H^{I}$ is the Hamiltonian for the inner space. The subscript $o$ denotes open channels for the scattering system described by $H^{I}$. The merit of this formula is that one can divide $\sigma(E)$ into a smooth background signal $\sigma_{b g}$ and a fluctuating part of the photoionization cross section. The latter contains the information about doubly excited states and thus most of the resonance states making up the chaotic fluctuations in the cross section for $E \rightarrow 0^{-}$. Starting from Eq. (3), one can now derived the semiclassical results described in the introduction as detailed in $[17]$. 
Total photoionization cross section of planer helium: scaling laws and collision orbits 4

\subsection{Quantum mechanical treatment in hyperspherical coordinates}

The Hamiltonian of the helium atom with infinite nucleus mass fixed at the origin is written as

$$
H=\frac{\left(\mathbf{p}_{1}^{2}+\mathbf{p}_{2}^{2}\right)}{2}-\frac{Z}{r_{1}}-\frac{Z}{r_{2}}+\frac{1}{\left|\mathbf{r}_{1}-\mathbf{r}_{2}\right|},
$$

where $\mathbf{r}_{i}$ and $\mathbf{p}_{i}, i=1,2$, denote the positions and the conjugate momenta of electron $i$ respectively. $Z$ is the nuclear charge. We will consider in the following the planar helium atom restricted to the $\mathrm{x}-\mathrm{y}$ plane, that is, both electrons move in the $z=0$ plane. This is an invariant sub-space of the full classical dynamics for initial conditions $z_{i}=0$, $p_{z i}=0$ and is considered here as a lower-dimensional problem quantum mechanically exhibiting the proliferation of Rydberg-series typical for the full helium problem.

In hyperspherical coordinates $\left(R, \alpha, \theta_{1}, \theta_{2}\right)$ with $R=\sqrt{r_{1}^{2}+r_{2}^{2}}, \alpha=\tan ^{-1}\left(r_{2} / r_{1}\right)$, and $\theta_{1}$ and $\theta_{2}$, the polar angles with respect to the $x$-axis, the Schrödinger equation is written as

$$
\left(-\frac{1}{2} \frac{\partial^{2}}{\partial R^{2}}+H_{R}\right) \Psi=E \Psi
$$

with $\Psi(R, \Omega)=R^{3 / 2} \bar{\Psi}$. Here, $\bar{\Psi}$ is a solution of the Schödinger equation $(H-E) \bar{\Psi}=0$ and $H_{R}$ is the adiabatic Hamiltonian for angle variables $\Omega=\left(\alpha, \theta_{1}, \theta_{2}\right)$. The adiabatic Hamiltonian $H_{R}$ is written as

$$
H_{R}=\frac{1}{2 R^{2}}\left(\Lambda^{2}+\frac{3}{4}+2 R C\left(\alpha, \theta_{1}, \theta_{2}\right)\right),
$$

where

$$
\Lambda^{2}=-\frac{1}{\sin (2 \alpha)} \frac{\partial}{\partial \alpha}\left[\sin (2 \alpha) \frac{\partial}{\partial \alpha}\right]+\frac{\mathrm{l}_{1}^{2}}{\cos ^{2} \alpha}+\frac{\mathrm{l}_{2}^{2}}{\sin ^{2} \alpha}
$$

with azimuthal angular momentum operators of the electrons 1 and $2, \mathbf{l}_{1}=\frac{1}{i} \frac{\partial}{\partial \theta_{1}}, \mathbf{l}_{2}=$ $\frac{1}{i} \frac{\partial}{\partial \theta_{2}}$, and the potential $C$ is given as

$$
C(\alpha, \theta)=-\frac{Z}{\cos \alpha}-\frac{Z}{\sin \alpha}+\frac{1}{\sqrt{1-\sin (2 \alpha) \cos \theta}},
$$

where $\theta=\theta_{2}-\theta_{1}$. The total angular momentum is $\mathbf{l}=\mathbf{l}_{1}+\mathbf{l}_{2}$. The wave function $\Psi(R, \Omega)$ is expanded in terms of adiabatic channel basis functions $\Phi_{n}(\Omega ; R)$ in the form

$$
\Psi(R, \Omega)=\sum_{n} F_{n}(R) \Phi_{n}(\Omega ; R)
$$

where $\Phi_{n}(\Omega ; R)$ are the eigenfunctions of the adiabatic Hamiltonian, that is,

$$
H_{R} \Phi_{n}(\Omega ; R)=U_{n}(R) \Phi_{n}(\Omega ; R) .
$$

Substitution of the expansion Eq. (9) into the Schrödinger equation (5) produces a set of $N_{C H} \times N_{C H}$ coupled-channel equations about $F(R)$, where $N_{C H}$ is the number of channels included in Eq. (9). The coupled-channel equations are solved using the SVD generalized log-derivative propagation method, called L-matrix propagation $[17,22,23,24]$, which is highly suitable for parallel computation. The dividing surface 
Total photoionization cross section of planer helium: scaling laws and collision orbits 5

$\Sigma$ is taken at hyperradius $R_{0}=20$. Note that if $R_{0}$ is greater than the support of the initial wave function $\phi_{0}$, the TPICS results become independent of $R_{0}$. By solving the Schödinger equation for the half-space Hamiltonians, the scattering matrices $\mathbf{s}$, $\mathbf{r}$, and the dipole transition amplitude $\mathbf{d}$, necessary for Eq. (3), are obtained.

For the Hamiltonian (4), there are 5 symmetry operators, namely $\Pi_{x}, \Pi_{y}, \Pi, P_{12}$, $L_{z}$ [25]. Here, $\Pi_{x}$ and $\Pi_{y}$ are parity operators about the $x$ and $y$-axis respectively, $\Pi$ is a point reflection $\left(\mathbf{r}_{1} \rightarrow-\mathbf{r}_{1}, \mathbf{r}_{2} \rightarrow-\mathbf{r}_{2}\right), L_{z}$ coincides here with the total angular momentum operator, and $P_{12}$ is the particle exchange operator. The spectrum of $H$ are classified by taking the mutual commuting operators $P_{12}, \Pi_{x}$, and $L_{z}$,

For a given total angular momentum $l$ the adiabatic function $\Phi(\Omega ; R)$ can be expanded as $\Phi_{l}(\Omega ; R)=\sum_{m} \Phi_{m}(\alpha ; R) e^{i\left((l-m) \theta_{1}+m \theta_{2}\right)}$, where $\Phi_{m}(\alpha ; R)$ is a function of $\alpha$ at fixed $R$. By rearranging the basis, we can write the function $\Phi_{l}(\Omega ; R)$ in terms of fixed quantum numbers also for of $\Pi_{x}$ and $P_{12}$. For example, the triplet states with $\Pi_{x}=1, P_{12}=1$ and $l=1$ or $l=0$ can be written as

$$
\begin{aligned}
\Phi_{l=1}^{\text {triplet }}(\Omega ; R)=\sum_{m} & {\left[A_{m}^{-}(\alpha ; R) \cos (\bar{\theta}) \cos \left(m-\frac{1}{2}\right) \theta\right.} \\
+ & \left.A_{m}^{+}(\alpha ; R) \sin (\bar{\theta}) \sin \left(m-\frac{1}{2}\right) \theta\right],
\end{aligned}
$$

and

$$
\Phi_{l=0}^{\text {triplet }}(\Omega ; R)=\sum_{m} B_{m}^{+}(\alpha ; R) \cos (m \theta),
$$

respectively. Here $\bar{\theta}=\left(\theta_{2}+\theta_{1}\right) / 2$ and

$$
\begin{aligned}
& A_{m}^{ \pm}(\alpha ; R)=A_{m}(\alpha ; R) \pm A_{m}\left(\frac{\pi}{2}-\alpha ; R\right), \\
& B_{m}^{+}(\alpha ; R)=B_{m}(\alpha ; R)+B_{m}\left(\frac{\pi}{2}-\alpha ; R\right),
\end{aligned}
$$

where $A_{m}(\alpha ; R)$ and $B_{m}(\alpha ; R)$ are functions of $\alpha$ at a given $R$. We expand $A_{m}(\alpha ; R)$ and $B_{m}(\alpha ; R)$ in terms of B-spline basis function [26]. In this basis, the adiabatic Hamiltonian $H_{R}$ is represented by a banded matrix of large size, e.g., $9572 \times 1055$ for triple state in the range $I_{19}$ to $I_{20}$. We calculate the adiabatic potential $U_{n}(R)$ and the channel functions $\Phi_{n}(\Omega ; R)$ up to $N_{C H}$ by using the ARPACK package [27].

\section{Numerical Results}

The initial state is taken as the lowest lying triplet bound state, with angular momentum $l=0$ and $\Pi_{x}=+1$ as done in Ref. [21]. In order to get the initial state, we use the matching method introduced in Ref. [17] with the boundary conditions $F(0)=F\left(R_{0}\right)=$ 0 for $F(R)$ and by imposing the condition $\Phi_{n}\left(\pi / 2-\alpha, \theta_{1}, \theta_{2} ; R\right)=\Phi_{n}\left(\alpha, \theta_{1}, \theta_{2} ; R\right)$. As a searching algorithm. we use the bisection method for simplicity. For the parameters, $h=0.1, n b p s=100, M_{\max }=20$, and $N_{C H}=20$, the ground energy is obtained as $E_{0}=-8.295967$, which is very close to the value given in Ref. [21]. Here nbps is the 
Total photoionization cross section of planer helium: scaling laws and collision orbits 6

Table 1. Parameters used in the calculation of the TPICS. $h_{\text {inner }}$ and $h_{\text {outer }}$ denote the step size of the inner $\left(R<R_{0}\right)$ and outer region $\left(R>R_{0}\right)$ in the $R$ direction.

\begin{tabular}{rcccccc}
\hline \multicolumn{1}{c}{$N$} & $R_{\infty}$ & $N_{C H}$ & $h_{\text {inner }}$ & $h_{\text {outer }}$ & $n b p s$ & $M_{\max }$ \\
\hline $1-10$ & 1000 & 200 & 0.1 & 0.1 & 200 & 20 \\
$10-15$ & 3000 & 400 & 0.1 & 0.1 & 300 & 20 \\
$15-20$ & 6000 & 600 & 0.05 & 0.1 & 150 & 30 \\
$20-21$ & 6000 & 746 & 0.05 & 0.1 & 170 & 40 \\
$21-22$ & 6000 & 820 & 0.05 & 0.1 & 170 & 40 \\
\hline
\end{tabular}

number of B-spline basis functions for $\alpha, M_{\max }$ denotes the number of basis functions for $\theta$, and $h$ is the step size in the $R$ direction.

The final state $\Psi_{f}^{+}(E)$ is taken as the scattering state satisfying outgoing boundary conditions with $l=1, \Pi_{x}=+1$, and $P_{12}=1$ (triplet state). By employing the mixed L- and S-matrix propagation method [17], we obtain the scattering matrix $\mathbf{r}$ for the outer region $\left(R>R_{0}\right)$ and $\Psi_{f}^{+}(E)$ in the inner region $\left(R<R_{0}\right)$. The outgoing boundary condition for $\mathbf{r}$ is applied at a sufficiently large $R=R_{\infty}$. The dipole transition amplitude $\mathbf{d}$ is obtained using the $J$-integral algorithm [28] for parallel computation. The parameter values used are varied with the energy region considered as shown in Table 1. We used 500 CPUs in the KISTI supercomputing center [29]. As a test of the program, we calculate the lowest state energy of $l=1$ triplet state with $\Pi_{x}=1$ and $P_{12}=1$, which is given by -8.225774 for $h=0.05, R_{0}=100, M_{\max }=20, n b p s=200$, and $N_{C H}=200$. The value is in good agreement with Ref. [30]. Note that the state is different from the initial ground state mentioned above, of which the angular momentum is not $l=1$ but $l=0$.

In Fig. 1, the total photoionization cross section (TPICS) is presented as a function of $N$ for the energy region of $I_{1}$ to $I_{15}$, where $I_{N}$ and $N$ are related by $I_{N}=-2 /(N-1 / 2)^{2}$ for $Z=2$. We can see some peaks in the smooth part $\sigma_{b g}$ at low energies with $N<4$, which are related to resonant states localized in the region $R<R_{0}$. However, $\sigma_{b g}$ is smooth for $N>4$ and the high lying resonances are indeed all contained in the fluctuating part of $\sigma$ as expected. This is because the support of the resonant wave functions expands far beyond the boundary $R=R_{0}$ and their contribution to $\sigma_{b g}$ becomes negligible. $\sigma_{b g}$ converges to a constant value as $N$ increases. $\sigma_{f l}$, on the other hand, is highly fluctuating for $N>4$ with decreasing amplitude of fluctuation as $N$ increases. We note a slight overall increase of the average value of $\sigma_{f l}$ which is a remanent of the smooth part contributing to the $R>R_{0}$ region. Such a behavior has been also seen in the cross section $\sigma_{f l}$ of eZe collinear helium [17].

In Fig. 2, the TPICS and its fluctuating part are plotted from $I_{10}$ to $I_{22}$. Because of the non-zero average for $\sigma_{f l}$, a smooth part is subtracted directly from total the cross section $\sigma(E)$. This smooth part is obtained by fitting the function $f(N)=a+b / N^{2}$ to $\sigma(E)$ and subtracting it to obtain $\sigma_{f l}(E)$ as shown in Fig.2(b). We note again that the amplitude of the fluctuating signal decreases as $|E| \rightarrow 0$ from below. Hereafter, $\sigma_{f l}$ will 
Total photoionization cross section of planer helium: scaling laws and collision orbits 7

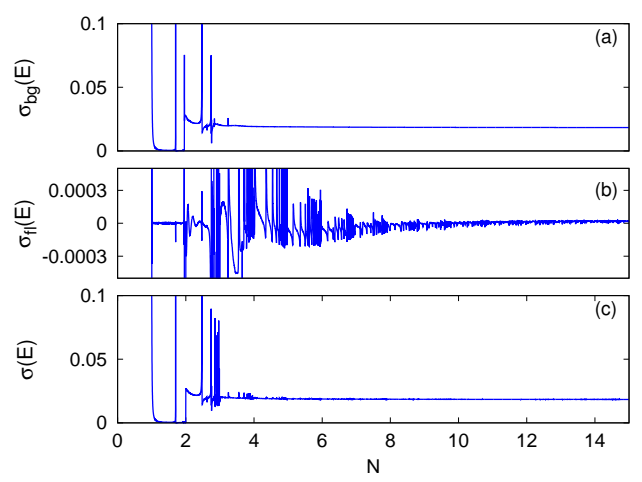

Figure 1. Total photoionization cross section $\sigma(E)$ of planar helium atom for $N=[1,15]$ as a function of $N(E)=\sqrt{2 /|E|}+1 / 2$. (a) the smooth part $\sigma_{b g}(E)$. (b) the fluctuating part $\sigma_{f l}(E)$. (c) the total cross section $\sigma(E)=\sigma_{b g}(E)+\sigma_{f l}(E)$. Here $\sigma_{b g}(E)$ and $\sigma_{f l}(E)$ mean what are defined in Eq. (3).

mean the fluctuation part obtained by the above fitting method.

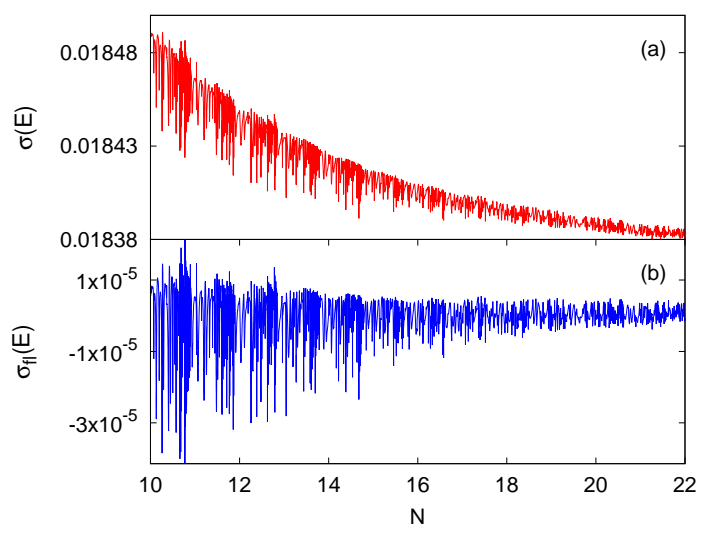

Figure 2. (a) Total photoionization cross section for range $N=[10,22]$ and (b) its fluctuating part $\sigma_{f l}(E)$. The smooth part is here obtained by fitting the function $f(N)=a+b / N^{2}$ to the signal in (a).

In order to confirm the semiclassical prediction (i) stated in Sec. 1, we calculate the Fourier transform (FT) of the signal in the from

$$
G(S)=\int F(z) e^{i S z} d z
$$

with $F(z)$ defined as

$$
F(z)=\frac{1}{4 \pi \alpha\left(E-E_{0}\right)|E|^{\mu}} \sigma_{f l}(z),
$$

where $z=1 / \sqrt{|E|}, \mu$ is the scaling exponent given in Eq. (1) with $\mu=1.306$ for $Z=2$, and $E_{0}$ is the energy of the initial state. The factor $|E|^{-\mu}$ is introduced to compensate 
for the decrease in amplitude. In Fig. 3, the Fourier transform of $F(z)$ with respect to $z$ is plotted as a function of the scaled variable $\tilde{S}=S \sqrt{|E|}$; note that the variable $S$ can be identified with a classical action. When comparing the position and height of the peaks in the FT of the total cross sections (obtained from a full quantum calculation) with the semiclassical results deduced from the actions and stabilities of closed triple collision orbits (CTCO) shown as blue circles, one finds excellent agreement. The position of the circles represents the scaled classical action of a specific CTCO whose trajectory is shown as an inset above the peak in Fig. 3. The heights are obtained by considering the stability matrix of the orbit as described in [17]. In the figure, the relative heights are plotted. For the range below $\tilde{S} /(2 \pi) \approx 8.6$ we can see that there is a one to one correspondence between the peaks of the quantum results and the positions of the classical actions of CTCO's. For larger actions, the correspondence deteriorates due to the finite width of the quantum signal $\sigma(E)$ and thus finite resolution of the FT. We expect that further peaks will be resolved if the TPICS is obtained for higher $N$ such as has been demonstrated in the lower dimensional eZe collinear helium in [16, 17]; here TPICS data were obtained up to $N=50$ and peaks were well resolved up to $\tilde{S} /(2 \pi) \approx 15$. The FT result for planar helium is almost identical to one for eZe collinear helium, except for the resolution.

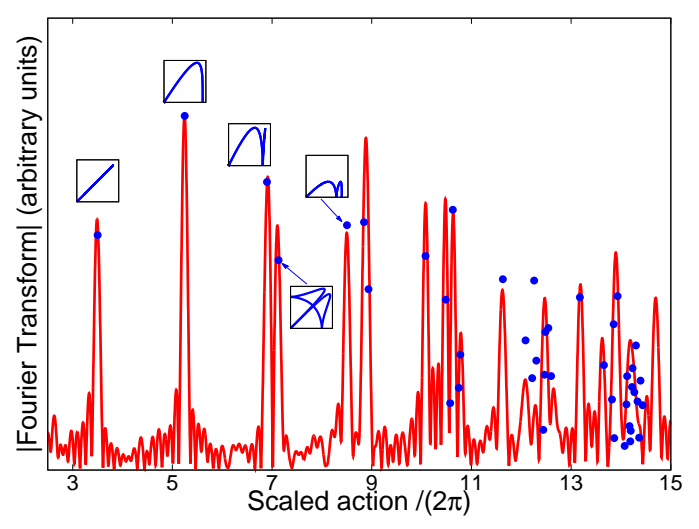

Figure 3. The Fourier spectrum of the fluctuating part of the cross section of planar helium rescaled according to Eq. (15); the position and height of the circles denote the action and (relative) size of the stability matrix of the corresponding CTCO's with $\tilde{S} / 2 \pi<15$. In the insets the CTCO trajectories in configuration space are shown for some of the peaks.

A validation of the scaling law, the second semiclassical prediction in Sec. 1, is obtained by extracting a decay exponent $\mu$ from the strongly fluctuating signal shown in Fig. 2. This is a somewhat delicate task and is done here by fitting a decay law directly to the signal $\sigma_{f l}$ after suitable smoothing. In order to avoid artifacts due to the variation in curvature in the original signal $\sigma_{f l}(N)$ when using large averaging intervals, we average the absolute value of $\sigma_{f l}(N)$ over fixed intervals of size $\Delta N$, that is, we 
Total photoionization cross section of planer helium: scaling laws and collision orbits 9

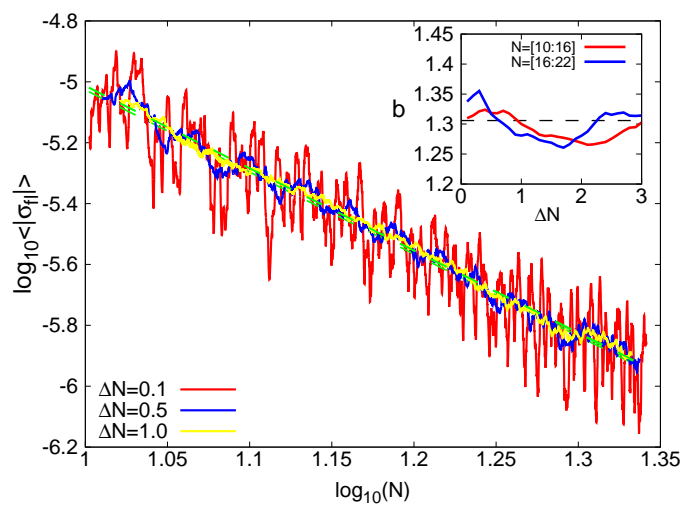

Figure 4. The averages of the absolute value of $\left|\sigma_{f l}\right|$ over intervals $\Delta N=0.1$ (red line), $\Delta N=0.5$ (blue line), and $\Delta N=1.0$ (yellow line) on a log-log scale; inset: extracted values of the decay exponent from three different sets of data $(N=[10,16]$ : red line: $N=[16,22]$ : blue line) and the expected value for planar $\mathrm{He}, \mu=1.306$ (dashed line).

consider

$$
\left\langle\left|\sigma_{f l}(N)\right|\right\rangle_{\Delta N}=\frac{1}{\Delta N} \int_{N-\Delta N / 2}^{N+\Delta N / 2}\left|\sigma_{f l}(N)\right| d N .
$$

The results are shown in Fig. 4 on a log-log scale, here for $\Delta N=0.1,0.5,1.0$. The smoothed data show a linear behavior on the log-log scale indicating a single-valued decay law. We extract the slope of the curve in Fig. 4 using a linear regression model with least-square fitting, that is, $\log _{10}\left\langle\left|\sigma_{f l}(N)\right|\right\rangle_{\Delta N} \approx a-2 b \log _{10}(N)$ with fit parameters $a$ and $b$. The parameter $b$ is compared with the theoretically predicted decay exponent $\mu$, see inset in Fig. 4. We would expect that $b$ is independent of $N$ if $N$ is sufficiently large, thus $b$ should be independent of the fitting range. We take two regions $N=[10,16]$ and $N=[16,22]$ from the whole region $N=[10,22]$, and then determined the slope of the averaged $\left|\sigma_{f l}\right|$ for each regions separately. The results for the fit parameter $b$ for each of these intervals are shown in the inset of Fig. 4 for different averaging intervals $\Delta N$. The data coincide with the expected value within $\pm 6 \%$, that is, we obtain an exponent of the order $\mu=1.3 \pm 0.08$.

\section{Conclusions}

By using an ab initio numerical method, the total photoionization cross section has been calculated for planar helium up to $I_{22}$ for the first time. The analysis of the fluctuating part of the TPICS confirms the semiclassical predictions in Ref. [16]. This is further strong evidence, that the fluctuation is completely dominated by the classical triple collision orbits all lying in the collinear eZe subspace embedded in the threedimensional space. Although an analysis of the full 3D helium problem is still out of reach both theoretically and experimentally due to the limitations in resolving the full spectrum for higher energies up to $N \approx 20$, this gives further confidence in expecting 
Total photoionization cross section of planer helium: scaling laws and collision orbits10

that the semiclassical predictions are also valid in the case of the full helium problem as well as other two-electron atoms.

\section{Acknowledgments}

This work was supported by the Institute for Basic Science under IBS-R012-D1.

\section{References}

[1] Madden R P and Codling K 1963 Phys. Rev. Lett. 1012516

[2] Baertschy M, Rescigno T N, Isaacs W A, Li X and McCurdy C W 2001 Phys. Rev. A 63022712

[3] Bartlett P L 2006 J. Phys. B: At. Mol. Opt. Phys. 39 R379

[4] Bray I and Stelbovics A T 1992 Phys. Rev. A 466995

[5] Bray I, Fursa D V, Kheifets A S and Stelbovics A T 2002 J. Phys. B: At. Mol. Opt. Phys. 35 R117

[6] Colgan J and Pindzola M S 2002 Phys. Rev. Lett. 8817173002

[7] Laulan S and Bachau H 2003 Phys. Rev. A 681013409

[8] Hu S X, Colgan J and Collins L A 2005 J. Phys. B: At. Mol. Opt. Phys. 38 L35

[9] Pindzola M S, Robicheaux F, Loch S D, Berengut J C, Topcu T, Colgan J, Foster M, Griffin D C, Ballance C P, Schultz D R, Minami T, Badnell N R, Witthoeft M C, Plante D R, Mitnik D M, Ludlow J A and Kleiman U 2007 J. Phys. B: At. Mol. Opt. Phys. 40 R39

[10] Feist J, Nagele S, Pazourek R, Persson E, Schneider B I, Collins L A and Burgdörfer J 2008 Phys. Rev. A 774043420

[11] Tanner G, Richter K and Rost J M 2000 Rev. Mod. Phys. 72497

[12] Hilico L, Grémaud B, Jonckheere T, Billy N and Delande D 2002 Phys. Rev. A 66022101

[13] Eiglsperger J and Madroñero J 2010 Phys. Rev. A 82033422

[14] Czasch A, Schöffler M, Hattass M, Schössler S, Jahnke T, Weber T, Staudte A, Titze J, Wimmer C, Kammer S, Weckenbrock M, Voss S, Grisenti R E, Jagutzki O, Schmidt L P H, SchmidtBöcking H, Dörner R, Rost J M, Schneider T, Liu C N, Bray I, Kheifets A S and Bartschat K 2005 Phys. Rev. Lett. 95243003

[15] Jiang Y H, Püttner R, Delande D, Martins M and Kaindl G 2008 Phys. Rev. A 78021401

[16] Byun C W, Choi N N, Lee M H and Tanner G 2007 Phys. Rev. Lett. 98113001

[17] Lee M H, Byun C W, Choi N N and Tanner G 2010 Phys. Rev. A 81043419

[18] Wannier G H 1953 Phys. Rev. 90817

[19] Tanner G, Choi N N, Lee M H, Czasch A and Dörner R 2007 J. Phys. B: At. Mol. Opt. Phys. 40 F157

[20] Buchleitner A, Gremaud B and Delande D 1994 J. of Phys. B: At., Mol. Opt. Phys. 272663

[21] Eiglsperger J and Madroñero J 2009 Phys. Rev. A 80022512

[22] Mrugała F and Secrest D 1983 J. Chem. Phys. 785954

[23] Mrugała F and Secrest D 1983 J. Chem. Phys. 795960

[24] Mrugała F 1993 Int. Rev. Phys. Chem. 121

[25] Madroñero J and Buchleitner A 2008 Phys. Rev. A 77053402

[26] Bachau H, Cormier E, Decleva P, Hansen J E and Martín F 2001 Rep. Prog. Phys. 641815

[27] Lehoucq R, Sorensen D and Yang C 1997 ARPACK: Solution of Large Scale Eigenvalue Problems by Implicitly Restarted Arnoldi Methods

[28] Mrugala F 1989 J. Chem. Phys. 91874

[29] http://www.kisti.re.kr

[30] Madroñero J 2004 Spectral properties of planar helium under periodic driving Dissertation, LudwigMaximilians-Universität München (Preprint http://edoc.ub.uni-muenchen.de/archive/ 00002187) 